\title{
Shape Mixing and Clustering in Nuclei: Probing Physics beyond the Standard Model
}

\author{
G.H. Sargsyan ${ }^{1}$, K.D. Launey ${ }^{1}$, A. Mercenne ${ }^{2}$, T. Dytrych ${ }^{1,3}$, \\ J.P. Draayer ${ }^{1}$ \\ ${ }^{1}$ Department of Physics and Astronomy, Louisiana State University, \\ Baton Rouge, LA 70803, USA \\ ${ }^{2}$ Center for Theoretical Physics, Sloane Physics Laboratory, Yale University, \\ New Haven, Connecticut 06520, USA \\ ${ }^{3}$ Nuclear Physics Institute of the Czech Academy of Sciences, \\ 25068 Řež, Czech Republic
}

Received 30 November 2021

doi: https://doi.org/10.55318/bgjp.2022.49.1.047

\begin{abstract}
The $a b$ initio methods in nuclear physics play a major role due to their use of realistic internucleon interactions that grant them predictive capabilities. One of these models - the symmetry-adapted nocore shell model (SA-NCSM) - is capable of reaching the medium-mass region of the chart of the nuclides, by exploiting the emergent symmetries of nuclei, and is therefore well-suited for studying collective correlations and beta decay modes. We apply the SA-NCSM to calculate beta-decay observables essential to probe physics beyond the Standard Model. Our calculations of higher-order recoil terms in the beta decay of ${ }^{8} \mathrm{Li}$ help to significantly reduce the uncertainties in high-precision experiments that study the vector minus axial vector $(\mathrm{V}-\mathrm{A})$ structure of the weak interaction. Furthermore, we calculate neutrinoless double beta decay matrix elements important for determining whether the neutrino is its own antiparticle.
\end{abstract}

KEY WORDS: Ab initio methods, Neutrinoless double beta decay, Physics beyond the Standard Model.

\section{Introduction}

An appealing problem related to the conventional beta-decay processes is the existence of tensor currents in the weak interactions. The theory of $\beta$ decays assumes the vector minus axial vector $(\mathrm{V}-\mathrm{A})$ nature of interaction to satisfy the observed violation of the conservation of parity. This is embedded in the Standard Model of particle physics. So while the V-A theory remains consistent with all data, there remain strong incentives to look for divergences from 
$\mathrm{V}-\mathrm{A}$ nature in $\beta$ decays. In short, despite outstanding successes of the Standard Model, for many theoretical reasons, particularly those due to the number of undetermined parameters associated with the models used to describe the underpinning structure, the existence of new physics remains an expectation.

Various extensions to beyond the Standard Model (BSM) permit scalar, tensor and pseudoscalar interactions to arise [1]. Motivated by this, several experiments attempt to measure the possible effects of these interactions. In a recent experiment on ${ }^{8} \mathrm{Li} \beta$ decay [2] at Argonne National Laboratory the upper limit on the tensor coupling constant was set to $\left|C_{T} / C_{A}\right|^{2}<0.011$, where $C_{T}$ and $C_{A}$ are the tensor and axial vector coupling constants. The results were consistent with a purely $\mathrm{V}-\mathrm{A}$ interaction. However, one of the largest uncertainties in the experimental analysis comes from the recoil-order terms in the ${ }^{8} \mathrm{Li} \beta$ decay. These terms were previously experimentally deduced in Ref. [3], albeit with large uncertainties. We provided more precise predictions of these recoil-order terms in Ref. [4], hence improving the constraints on the tensor force in the weak interaction, by performing $a b$ initio calculations using the symmetry-adapted no-core shell model (SA-NCSM) [5,6]. In the current work we discuss additional results that were vital in determining the values of the beta decay recoil-order terms.

Another promising approach for studying physics beyond the Standard Model is neutrinoless double-beta decay $(0 \nu \beta \beta)$. The discovery of this phenomenon and its further study could shed light on some of the most profound questions in nuclear physics, particle physics, astrophysics and cosmology. In particular, the observation of $0 \nu \beta \beta$ would provide direct evidence that the neutrinos are Majorana particles, as well as for a BSM process that violates lepton number conservation and constrains the neutrino mass scale [7].

To offer deeper insight into the nuclear matrix elements of $0 \nu \beta \beta$, novel ab initio models are essential. Although often constrained to comparatively light nuclei by the exponential growth of the model space with the increasing number of nucleons, $a b$ initio models are powerful tools for studying nuclear properties. Recent results from ab initio studies have indicated the need for further improvements [8]. Namely, while the coupled-cluster theory [9] can provide a successful description of ${ }^{48} \mathrm{Ca}$, calculating the $0 \nu \beta \beta$ rate to ${ }^{48} \mathrm{Ti}$ is extremely challenging; another $a b$ initio model, the in-medium similarity renormalization group (IMSRG) [10], uses highly renormalized interactions that might affect the final result. The SA-NCSM has been used to describe the structure of ${ }^{48} \mathrm{Ti}$ [11] and can be employed without the need for using effective operators and charges for calculations of the lightest $0 \nu \beta \beta$ candidate, ${ }^{48} \mathrm{Ca}$.

\section{$2{ }^{8}$ Li Beta Decay}

The recoil-order terms are usually omitted in $\beta$-decay considerations since they are of the order of $q / m_{N}$ or higher, where $q$ is the momentum transfer (typ- 
ically several $\mathrm{MeV} / \mathrm{c}$ ) and $m_{N}$ is the nucleon mass. Hence, for most decays, the recoil effects contribute less than a percent compared to the dominant Fermi and Gamow-Teller (GT) terms. Yet, for the cases of precise allowed $\beta$ spectra measurements, these terms must be included in the analysis. The $\beta$ decay recoilorder terms have been derived using the impulse approximation in Ref. [12]. We consider the second forbidden axial vector $\left(j_{2}\right.$ and $\left.j_{3}\right)$ terms, since they account for the recoil-order corrections and their comparatively large uncertainties in the measurements of weak interaction tensor currents due to the relatively large error bars on them measured by Ref. [3]. In the impulse approximation the $j_{2,3}$ terms along with the GT $\left(c_{0}\right)$ are given as:

$$
\begin{aligned}
c_{0}\left(q^{2}\right) & =(-)^{\left(J^{\prime}-J\right)} \frac{g_{A}\left(q^{2}\right)}{\sqrt{2 J+1}}\left\langle J^{\prime}|| \sum_{i=1}^{A} \tau_{i}^{ \pm} \sigma_{i}|| J\right\rangle \\
& =(-)^{\left(J^{\prime}-J\right)} \frac{g_{A}\left(q^{2}\right)}{\sqrt{2 J+1}} M_{G T}, \\
j_{K}\left(q^{2}\right) & =-(-)^{\left(J^{\prime}-J\right)} \frac{2}{3} \frac{g_{A}\left(q^{2}\right)}{\sqrt{2 J+1}} \frac{\left(A m_{N} c^{2}\right)^{2}}{(\hbar c)^{2}} \\
& \times\left\langle J^{\prime}|| \sum_{i=1}^{A} \tau_{i}^{ \pm}\left[Q_{i} \times \sigma_{i}\right]^{K}|| J\right\rangle, \text { with } K=2,3,
\end{aligned}
$$

where $m_{N}$ is the nucleon mass $g_{V}(0)=1$ and $g_{A}(0) \approx 1.27$ are the vector and axial coupling constants, $A$ is the mass number and $J$ and $J^{\prime}$ are the total angular momenta of the initial and final nuclei, respectively. $M_{G T}$ is the conventional GT matrix element. The $\tau_{i} / 2$ and $\sigma_{i} / 2$ are the isospin and intrinsic spin operators, and $Q_{i}=\sqrt{16 \pi / 5} r_{i}^{2} Y_{2 \mu}\left(\hat{r}_{i}\right)$, is the quadrupole moment operator, of the $i^{\text {th }}$ particle.

The recoil-order form factors are generally reported as ratios $j_{2,3} / A^{2} c_{0}$. Prediction of this ratio require calculations of $c_{0}$ or the GT matrix element in the denominator. For the ${ }^{8} \mathrm{Li}$ ground state $\left(2^{+}\right) \beta$ decay there is only one final $2^{+}$state available in the currently accepted experimental energy spectrum of the ${ }^{8} \mathrm{Be}$. However, low-lying intruder $2^{+}$state has been proposed by Barker in Ref [13] through R-matrix analysis of experimental data, which also would be available as a beta-decay final state. Our calculations also indicate the existence of such state, which has important implications for precision measurements of ${ }^{8} \mathrm{Li}$ and ${ }^{8} \mathrm{~B} \beta$ decays. The calculated energy spectrum of ${ }^{8} \mathrm{Be}$ along with the $j_{2} / A^{2} c_{0}$ and $j_{3} / A^{2} c_{0}$ predictions can be found in Ref. [4].

It is important to note that the inclusion of a low-lying $2^{+}$state in the R-matrix analysis of $\beta$ decay data considerably changes the value of the GT matrix element. The extracted $M_{\mathrm{GT}}$ according to Ref. [15] shows that for a decay to the lowest $2^{+}$state in ${ }^{8} \mathrm{Be}$ decreases by almost 1.5 times when a low-lying $2^{+}$ intruder state is added. Such an analysis was also done by Barker in Ref. [16]. The extracted GT matrix element from that work is in a closer agreement with 
G.H. Sargsyan, K.D. Launey, A. Mercenne, T. Dytrych, J.P. Draayer

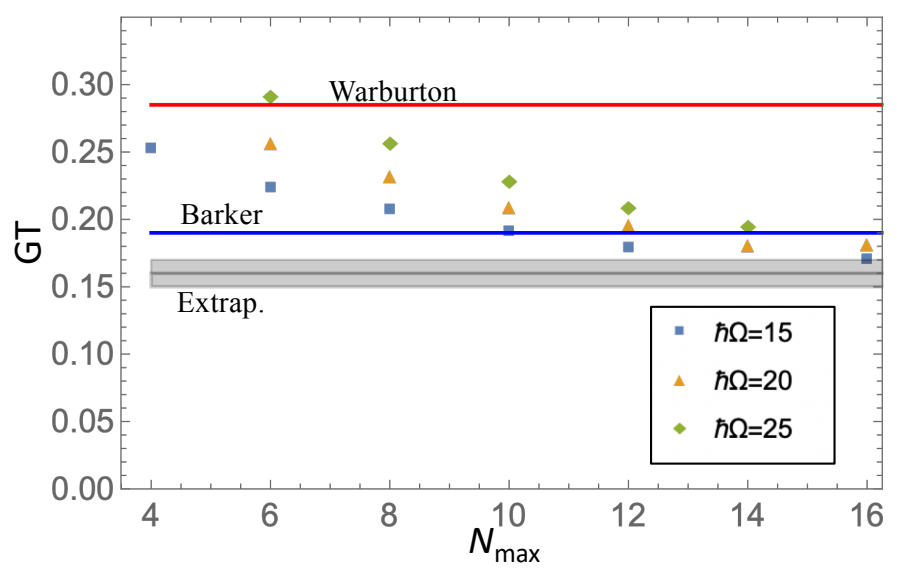

Figure 1. SA-NCSM calculations of GT matrix element for ${ }^{8} \mathrm{Li} \beta$ decay to the ${ }^{8} \mathrm{Be}$ lowest $2^{+}$state for various model spaces, and $\hbar \Omega=15,20$ and $25 \mathrm{MeV}$ using NNLO $_{\text {opt }}$ [14] interaction, and compared to the experimentally deduced values. The result from Warburton [15] does not include a low-lying intruder state, while the value from Barker [16] does. The gray band denoted by "Extrap." indicates extrapolated value from calculations with uncertainties.

the SA-NCSM $M_{\mathrm{GT}}$ compared to the one where no intruder $2^{+}$state was included (see Figure 1). Thus, our calculations provide a further support towards the existence of intruder states in the low-lying ${ }^{8} \mathrm{Be}$ spectrum.

The energies from Barker's R-matrix fits for the intruder $0^{+}$and $2^{+}$states are $\sim 6 \mathrm{MeV}$ and $9 \mathrm{MeV}$, respectively, with $\alpha$ widths $>7 \mathrm{MeV}$ since the energy available for an $\alpha$ particle decay is large. These excitation energies agree with the SA-NCSM extrapolated results given the error bars [4], as well as with the predicted widths. In addition, a recent experimental study has found a tentative evidence for a broad $0^{+}$state at $12 \mathrm{MeV}$, prompting a need for further measurements [17]. Various earlier ab initio studies have seen intruder $0^{+}$and $2^{+}$states as well [18-20]. In particular, the extrapolations in the earlier study in Ref. [18] predicted the intruder $0^{+}$state to be at $12 \mathrm{MeV}$ with an uncertainty of almost $5 \mathrm{MeV}$. It also predicted the corresponding $2^{+}$and $4^{+}$states from the same rotational band. Moreover, in the recent calculations with the Daejon16 NN interaction in Ref. [20] the energy of the intruder $0^{+}$is shown to drop to 11.71 $\mathrm{MeV}$ in the largest model space with $\hbar \Omega=15 \mathrm{MeV}$. The study also calculated the widths of the $0^{+}$and $2^{+}$states to be $8.86 \mathrm{MeV}$ and $3.57 \mathrm{MeV}$, respectively, using NCSM combined with the resonating group method (RGM). 


\section{Neutrinoless Double Beta Decay in Light Nuclei}

Double-beta decay is the process in which two electrons or two positrons are emitted from a nucleus. Such processes occur when the energy of a nucleus with odd protons and odd neutrons is higher than the energies of both of its neighboring even-even nuclei (e.g., see red arrow in Figure 2). Since protonneutron pairing correlations can explain this even-odd energy difference, it has been suggested that proton-neutron pairs play important role in calculating the double-beta decay rate. Double-beta decays are products of second-order perturbation induced by weak interaction and, therefore, have much smaller rates of occurrence or much longer half-lives $\left(T_{1 / 2}>10^{20}\right.$ years $)$ than single $\beta$ decays.

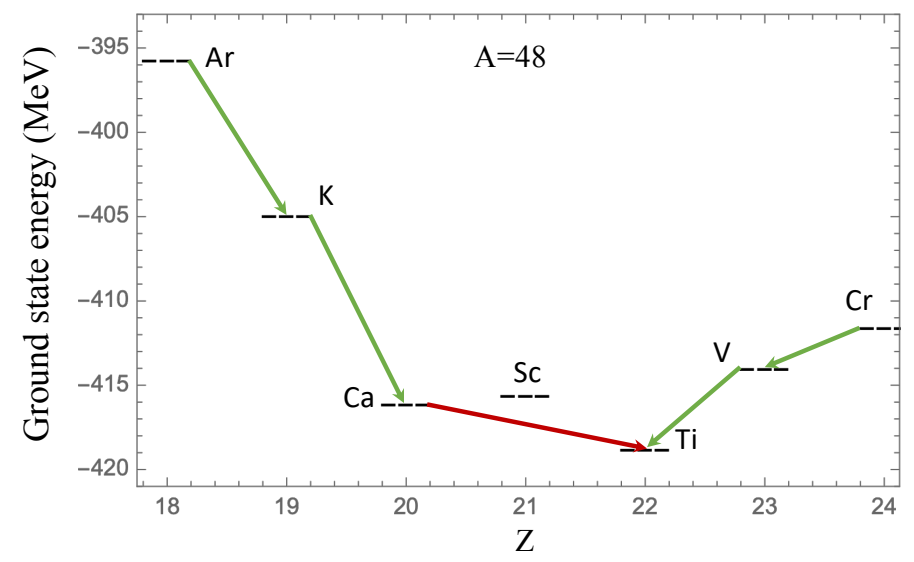

Figure 2. The experimental ground state energies of $A=48$ nuclei (isobaric chain). The green arrows represent single $\beta$ decay, and the red arrow displays the double-beta decay.

Double-beta decays with emission of two neutrinos (antineutrinos) have been detected for a number of nuclei. Nevertheless, one of the profound questions in modern physics is whether the double-beta decay can take place without emitting neutrinos. If neutrinos are Majorana particles (meaning there is no distinction between neutrino and antineutrino) and have a non-zero mass, then the neutrinoless double-beta decay can be imagined as absorption of the neutrino from the first $\beta$-decay in the intermediate state which induces the ejection of the second charged lepton.

As mentioned above, the process of neutrinoless double-beta decay is of great importance in the searches of BSM physics. Various collaborations around the world are performing experiments towards measuring the rate of this process in a number of nuclei. In order to provide guidance for the state-of-the-art experiments, theoretical calculations prove to be crucial.

The calculations of $0 \nu \beta \beta$ nuclear matrix element, $M_{0 \nu}$, are extremely challenging since there is no experimental data that can be used to validate the results 
from various models. To compare different $a b$ initio methods, benchmark calculations of $0 \nu \beta \beta$ for decays that are energetically forbidden or happen in competition with single $\beta$ decay have been performed for a number of light nuclei. In Refs. [21,22], the nuclear matrix elements for ${ }^{6,8,10} \mathrm{He}$ and ${ }^{10,12} \mathrm{Be}$ were calculated with a quantum Monte Carlo (QMC) approach based on the AV18 interaction. Later, the same calculations were carried out with the local Norfolk chiral interaction for ${ }^{6} \mathrm{He}$ and ${ }^{12} \mathrm{Be}$ [23]. In Ref. [24] the NCSM calculations of ${ }^{6} \mathrm{He}$ $M_{0 \nu}$ were benchmarked against the results from the multireference in-medium similarity renormalization group (MR-IMSRG) with a softened chiral interaction. Moreover, the $M_{0 \nu}$ of ${ }^{6,8,10} \mathrm{He},{ }^{14} \mathrm{C}$ and ${ }^{22} \mathrm{O}$ were calculated with the NCSM and coupled-cluster theory in Ref. [9]. For most of these nuclei along with ${ }^{10} \mathrm{Be}$, Ref. [25] calculated nuclear matrix elements with the importancetruncated (IT) NCSM [26] and two variants of IMSRG - the valence-shell (VS) IMSRG [27] and the in-medium generator coordinate method (IM-GCM) [28].

To benchmark the SA-NCSM results of $0 \nu \beta \beta$ against other methods, we perform calculations of $M_{0 \nu}$ for the light ${ }^{6} \mathrm{He} \rightarrow{ }^{6} \mathrm{Be}$ and intermediate-mass ${ }^{22} \mathrm{O}$ $\rightarrow^{22} \mathrm{Ne}$ ground-state-to-ground-state decays. For both of these systems we use the $\mathrm{NNLO}_{\text {opt }}$ interaction, which was not renormalized, as compared to the SRG evolved N3LO-EM interaction used in Refs. [9,24,25]. In the case of the $A=6$ system, the calculations are done up to $N_{\max }=8$ model space with $\hbar \Omega=20$ $\mathrm{MeV}$. This value of $\hbar \Omega$ parameter was shown to be optimal for the convergence of energies in Ref. [29] for $A=6$ systems and was used in NCSM calculations of Ref. [24] against which we benchmark our calculations. The results show that the GT matrix element is about four times larger than the Fermi contribution, whereas the tensor contribution is approximately two orders of magnitude smaller and of opposite sign (Figure 3). Moreover, it is important to note that these matrix elements show a fast convergence as the model space increases. We use the three-parameter exponential formula of Ref. [29] to extrapolate the total
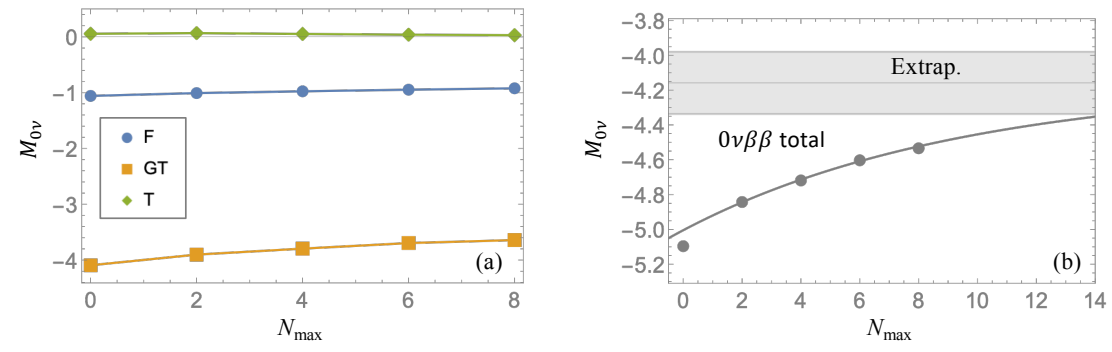

Figure 3. (a) Fermi (F), Gamow-Teller (GT) and Tensor (T) components and (b) total neutrinoless double-beta decay nuclear matrix element for the ${ }^{6} \mathrm{He} \rightarrow{ }^{6} \mathrm{Be}$ decay. The discrete points represent results of many-body calculations while lines represent fits. The gray band indicates the extrapolation uncertainty. Calculations are done using $\mathrm{NNLO}_{\mathrm{opt}}$ interaction with $\hbar \Omega=20 \mathrm{MeV} \mathrm{HO}$ parameter. 


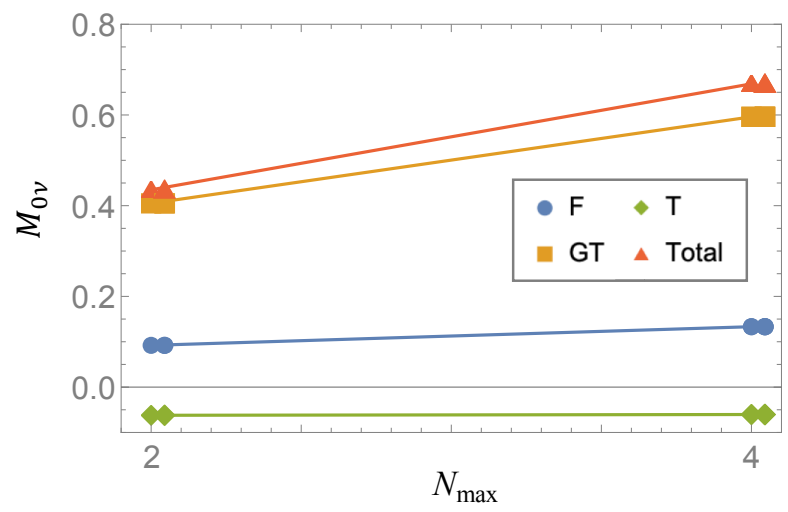

Figure 4. Fermi (F), Gamow-Teller (GT) and Tensor (T) components and the total neutrinoless double-beta decay nuclear matrix element for the ${ }^{22} \mathrm{O} \rightarrow{ }^{22} \mathrm{Ne}$ decay between the ground states. Calculations are done using NNLO $_{\text {opt }}$ interaction with $\hbar \Omega=15 \mathrm{MeV}$ HO parameter.

$M_{0 \nu}$, which yields $M_{0 \nu}=-4.16 \pm 0.18$. The results are in agreement with predictions from Refs. [9, 24, 25].

Both of the nuclei invlolved in the ${ }^{22} \mathrm{O} \rightarrow{ }^{22} \mathrm{Ne}$ decay have enhanced collectivities as indicated by their large $B(E 2)$ values [30], which has been a challenge to reproduce for $a b$ initio models. The SA-NCSM is able to provide an accurate description of collective correlations in this mass region as shown in Ref. [31]. Our calculations for the ${ }^{22} \mathrm{O} \rightarrow{ }^{22} \mathrm{Ne}$ decay performed with $\hbar \Omega=15 \mathrm{MeV}$ show an increase of the nuclear matrix element with $N_{\max }$ (Figure 4). This value of $\hbar \Omega$ was shown to be optimal for this mass region in Refs [31] and [32]. We note that in the IT-NCSM results from Ref. [25] all components of the nuclear matrix element stay practically the same as the model space increases.

\section{Conclusions}

In this work we calculated electromagnetic and weak transitions in nuclei from first principles. The results are valuable for helping probe physics beyond the Standard Model. The ab initio SA-NCSM was used to determine the size of the recoil-order form factors in the $\beta$ decay of ${ }^{8} \mathrm{Li}$. It showed that states of the $\alpha+\alpha$ system not included in the evaluated ${ }^{8} \mathrm{Be}$ energy spectrum have an important effect on all recoil-order terms, and can explain the $M_{\mathrm{GT}}$ discrepancy between the two experimental values in the $A=8$ system. In addition, we applied the symmetry-adapted basis to calculate ${ }^{6} \mathrm{He} \rightarrow{ }^{6} \mathrm{Be}$ and ${ }^{22} \mathrm{O} \rightarrow{ }^{22} \mathrm{Ne}$ neutrinoless double beta decay matrix elements. The results validate our method allowing us to perform further calculation on ${ }^{48} \mathrm{Ca}$ that is the lightest candidate of neutrinoless double beta decay used in experiments. 
G.H. Sargsyan, K.D. Launey, A. Mercenne, T. Dytrych, J.P. Draayer

\section{Acknowledgements}

We thank Saori Pastore and Garret King for useful discussions, as well as Jonathan Engel for providing the neutrinoless double beta decay matrix elements in the shell model basis. This work was supported in part by the U.S. National Science Foundation (PHY-1913728), U.S. Department of Energy (SC0019521) and SURA. It benefited from high performance computational resources provided by LSU (www.hpc.lsu.edu), the National Energy Research Scientific Computing Center (NERSC), a U.S. Department of Energy Office of Science User Facility operated under Contract No. DE-AC02-05CH11231, as well as the Frontera computing project at the Texas Advanced Computing Center, made possible by National Science Foundation award OAC-1818253.

\section{References}

[1] P. Herczeg (2001) Beta decay beyond the standard model. Prog. Part. Nucl. Phys. 46(2) 413-457.

[2] M.G. Sternberg, R. Segel, N.D. Scielzo, G. Savard, J.A. Clark, P.F. Bertone, F. Buchinger, M. Burkey, S. Caldwell, A. Chaudhuri, et al. (2015) Limit on tensor currents from ${ }^{8} \mathrm{Li} \beta$ decay. Phys. Rev. Lett. 115182501.

[3] T. Sumikama, K. Matsuta, T. Nagatomo, M. Ogura, T. Iwakoshi, Y. Nakashima, H. Fujiwara, M. Fukuda, M. Mihara, K. Minamisono, et al. (2011) Test of the conserved vector current hypothesis by a $\beta$-ray angular distribution measurement in the mass-8 system. Physical Review C 83065501.

[4] G.H. Sargsyan, K.D. Launey, M.T. Burkey, A.T. Gallant, N.D. Scielzo, G. Savard, A. Mercenne, T. Dytrych, D. Langr, L. Varriano, et al. (2021) Impact of clustering on the ${ }^{8} \mathrm{Li} \beta$ decay and recoil form factors. arXiv preprint arXiv:2107.10389, submitted to Phys. Rev. Lett.

[5] K.D. Launey, T. Dytrych, J.P. Draayer (2016) Symmetry-guided large-scale shellmodel theory. Prog. Part. Nucl. Phys. 89101 (review).

[6] T. Dytrych, K.D. Launey, J.P. Draayer, D.J. Rowe, J.L. Wood, G. Rosensteel, C. Bahri, D. Langr, R.B. Baker (2020) Physics of nuclei: Key role of an emergent symmetry. Phys. Rev. Lett. 124042501.

[7] J. Engel, J. Menendez (2017) Status and future of nuclear matrix elements for neutrinoless double-beta decay: a review. Rep. Prog. Phys. 80(4) 046301.

[8] Jonathan Engel (2017) Private communication.

[9] S. Novario, P. Gysbers, J. Engel, G. Hagen, G.R. Jansen, T.D. Morris, P. Navratil, T. Papenbrock, S. Quaglioni (2021) Coupled-cluster calculations of neutrinoless double- $\beta$ decay in ${ }^{48}$ Ca. Phys. Rev. Lett. 126182502. DOI: 10.1103/PhysRevLett.126.182502.

[10] J.M. Yao, B. Bally, J. Engel, R. Wirth, T.R. Rodriguez, H. Hergert (2020) Ab Initio Treatment of Collective Correlations and the Neutrinoless Double Beta Decay of ${ }^{48}$ Ca. Phys. Rev. Lett. 124 232501. DOI: 10.1103/PhysRevLett.124.232501.

[11] K.D. Launey, A. Mercenne, T. Dytrych (2021) Nuclear Dynamics and Reactions in the Ab Initio Symmetry-Adapted Framework. Annu. Rev. Nucl. Part. Sci. $71253-$ 277. 


\section{Probing Physics beyond the Standard Model}

[12] B.R. Holstein (1974) Recoil effects in allowed beta decay: The elementary particle approach.Rev. Mod. Phys. 46 789-814.

[13] F.C. Barker. (1969) $2^{+}$states of ${ }^{8}$ Be. Aust. J. Phys. 22 293-316.

[14] A. Ekström, G. Baardsen, C. Forssén, G. Hagen, M. Hjorth-Jensen, G.R. Jansen, R. Machleidt, W. Nazarewicz, et al. (2013) An optimized chiral nucleon-nucleon interaction at next-to-next-to-leading order. Phys. Rev. Lett. 110192502.

[15] E.K. Warburton (1986) R-matrix analysis of the $\beta^{ \pm}$-delayed alpha spectra from the decay of ${ }^{8} \mathrm{Li}$ and ${ }^{8} \mathrm{~B}$ Phys. Rev. C 33 303-313.

[16] F.C. Barker (1989) Delayed alpha spectra from the beta decay of ${ }^{8} \mathrm{Li}$ and ${ }^{8} \mathrm{~B}$. Aust. J. Phys. 42(1) 25-40.

[17] M. Munch, O.S. Kirsebom, J.A. Swartz, K. Riisager, H.O.U. Fynbo (2018) Measurement of the full excitation spectrum of the ${ }^{7} \mathrm{Li}(p, \gamma) \alpha \alpha$ reaction at $441 \mathrm{keV}$. Phys. Lett. B 782 779-784.

[18] E. Caurier, P. Navratil, W.E. Ormand, J.P. Vary (2001) Intruder states in ${ }^{8}$ Be. Phys. Rev. C 64051301.

[19] P. Maris (2013) Ab initio calculations for Be-isotopes with JISP16. J. Phys.: Conference Series 445012035.

[20] D.M. Rodkin, Yu.M. Tchuvil'sky (2020) Description of alpha-clustering of ${ }^{8} \mathrm{Be}$ nucleus states in high-precision theoretical approach. Chin. Phys. C $\mathbf{4 4} 124105$.

[21] S. Pastore, J. Carlson, V. Cirigliano, W. Dekens, E. Mereghetti, R.B. Wiringa (2018) Neutrinoless double- $\beta$ decay matrix elements in light nuclei. Phys. Rev. $C$ 97014606

[22] X.B. Wang, A.C. Hayes, J. Carlson, G.X. Dong, E. Mereghetti, S. Pastore, R.B. Wiringa (2019) Comparison between variational Monte Carlo and shell model calculations of neutrinoless double beta decay matrix elements in light nuclei. Phys. Lett. B 798134974.

[23] V. Cirigliano, W. Dekens, J. de Vries, M.L. Graesser, E. Mereghetti, S. Pastore M. Piarulli, U. van Kolck, R.B. Wiringa (2019) Renormalized approach to neutrinoless double- $\beta$ decay. Phys. Rev. $C, \mathbf{1 0 0} 055504$

[24] R.A.M. Basili, J.M. Yao, J. Engel, H. Hergert, M. Lockner, P. Maris, J.P. Vary (2020) Benchmark neutrinoless double- $\beta$ decay matrix elements in a light nucleus. Phys. Rev. C 102014302.

[25] J.M. Yao, A. Belley, R. Wirth, T. Miyagi, C.G. Payne, S.R. Stroberg, H. Hergert J.D. Holt (2021) Ab initio benchmarks of neutrinoless double- $\beta$ decay in light nuclei with a chiral hamiltonian. Phys. Rev. C 103014315.

[26] R. Roth (2009) Importance truncation for large-scale configuration interaction approaches. Phys. Rev. C 79064324.

[27] S.R. Stroberg, H. Hergert, J.D. Holt, S.K. Bogner, A. Schwenk (2016) Ground and excited states of doubly open-shell nuclei from ab initio valence-space hamiltonians. Phys. Rev. C 93051301.

[28] J.M. Yao, B. Bally, J. Engel, R. Wirth, T.R. Rodríguez, H. Hergert (2020) Ab Initio Treatment of Collective Correlations and the Neutrinoless Double Beta Decay of ${ }^{48}$ Ca. Phys. Rev. Lett. 124232501.

[29] P. Maris, J.P. Vary, A.M. Shirokov (2009) Ab initio no-core full configuration calculations of light nuclei. Phys. Rev. C 79014308.

[30] M. Shamsuzzoha Basunia (2015) Nuclear data sheets for $A=22$. Nuclear Dato Sheets 127 69-190. 
[31] P. Ruotsalainen, J. Henderson, G. Hackman, G.H. Sargsyan, K.D. Launey, A. Saxena, P.C. Srivastava, S.R. Stroberg, T. Grahn, J. Pakarinen, G.C. Ball, et al. (2019) Isospin symmetry in B(E2) values: Coulomb excitation study of ${ }^{21} \mathrm{Mg}$. Phys. Rev. C 99051301.

[32] J. Williams, G.C. Ball, A. Chester, T. Domingo, A.B. Garnsworthy, G. Hackman, J. Henderson, R. Henderson, R. Krucken, A. Kumar, et al. (2019) Structure of ${ }^{28} \mathrm{Mg}$ and influence of the neutron $p f$ shell. Phys. Rev. C 100014322. 